\title{
ENSEÑANZA DEL SISMO EN MÉXICO DE LOS DÍAS 19 Y 20 DE SEPTIEMBRE DE 1985 (INFLUENCIA DE LAS CONDICIONES LOCALES DE LOS SUELOS EN EL RIESGO SÍSMICO)
}

\author{
MEXICO EARTHQUAKE ON 19 AND 20 SEPTEMBER 1985 (INFLUENCE OF \\ LOCAL CONDITIONS OF SOILS IN SEISMIC RISK)
}

\section{GABRIEL MORENO PECERO}

Universidad Nacional Autónoma de México, México. morenop@unam.mx

RESUMEN

Se hace una presentación sobre, un análisis y se dan conclusiones y recomendaciones, para considerar en la generación de Reglamentos de Construcción de zonas urbanas, todo ello relacionado con el riesgo sísmico.

PALABRAS CLAVE: riesgo sísmico, influencia de condiciones locales de los suelos.

\section{ABSTRACT}

A presentation is made on, an analysis and conclusions and recommendations are given, to consider in the generation of Construction Regulations of urban areas, all related to the seismic risk.

KEYWORDS: seismic risk, Influence of local soil conditions. 
INTRODUCCIÓN

El jueves, 19 de septiembre de 1985, a las 7 horas 19 minutos, en la ciudad de Mexico ocurrió un sismo de 8.1 grados de magnitud en escala de Richter; al día siguiente, hubo una réplica de magnitud menor; su impacto, en los posteriores minutos a la ocurrencia del primer sismo, fue desigual; los habitantes de la zona sur de la ciudad, no se percataron del efecto del sismo porque, salvo zonas muy localizadas, no ocasionó ninguna situación que permitiera darse cuenta de lo sucedido; en cambio los del centro de la ciudad, y los que habitaban algunas colonias o barrios vecinos a ese centro, estaban totalmente al tanto de la magnitud de la tragedia, cuantificada cuando después se supo que prácticamente habían colapsado al rededor de 1200 edificios, generando la muerte de casi 10.000 seres humanos.

La caracterización y zonificación geotécnica de la ciudad fueron evacuadas por ingenieros de la Universidad Nacional Autónoma de México, apoyados por una importante empresa mexicana de ingeniería. Los resultados de esta tarea permitieron la publicación del libro El Subsuelo de la Ciudad de México, cuyos autores Raúl J. Marsal y Marcos Mazari dividieron la ciudad en tres zonas: la zona del lago, la zona de transición y la zona de loma.

La zona del lago constituida por arcilla $(\mathrm{CH})$ con contenidos naturales de agua, promedios, del orden de los $350 \%$, con consistencia relativa tal que el suelo puede calificarse de muy deformable, pero además con una resistencia al esfuerzo cortante baja, del orden de 5 ton/ $\mathrm{m} 2$, en prueba de compresión triaxial rápida. Se tiene un estrato superior de esa arcilla (FAS) con espesores que varían de los 33 metros a más de 80 metros, los primeros precisamente en el mencionado centro de la Ciudad de México; subyaciendo a la $\mathrm{CH}$ aparece la llamada "primera capa dura" formada por limo, arena y algo de arcilla, con espesores del orden de 4 metros, debajo de la cual se tiene otra capa de arcilla (FAI), en general, con una consistencia ligeramente mayor a la de la FAS. La zona de transición formada por arenas, limo y arcillas en proporciones diversas constituyendo una formación regional que en cierta frecuencia se denomina "tepetate" y finalmente la zona de limos en que aparecen rocas ígneas, con algún grado de alteración.

La parte centro de ciudad de México corresponde a la zona del lago, mientras que la parte sur de la ciudad queda en las llamadas zona de transición y zona de lomas.

El sismo de septiembre de 1985 evidenció su mayor impacto en ciertas zonas, como las denominadas Tlatelolco, Roma Sur y Norte y en la Taxqueña; en esta última está próxima la frontera entre la zona del lago y la de transición.

A las pocas horas de transcurrido el sismo, el autor de este artículo caminó una gran longitud del llamado Paseo de la Reforma, una de las principales arterias de la ciudad, hasta llegar al Palacio de Minería, a unos pasos del Teatro Bellas Artes, y pudo observar, una serie de edificios colapsados;

Se evidenció en ellos una característica común, su arquitectura correspondía a la tradicional de paralelepípedo y el número de niveles variado, entre 12 y 15; entre ellos destacaron algunos, por el daño sufrido, que ocupaban predios limitados por calles que formaban esquina.
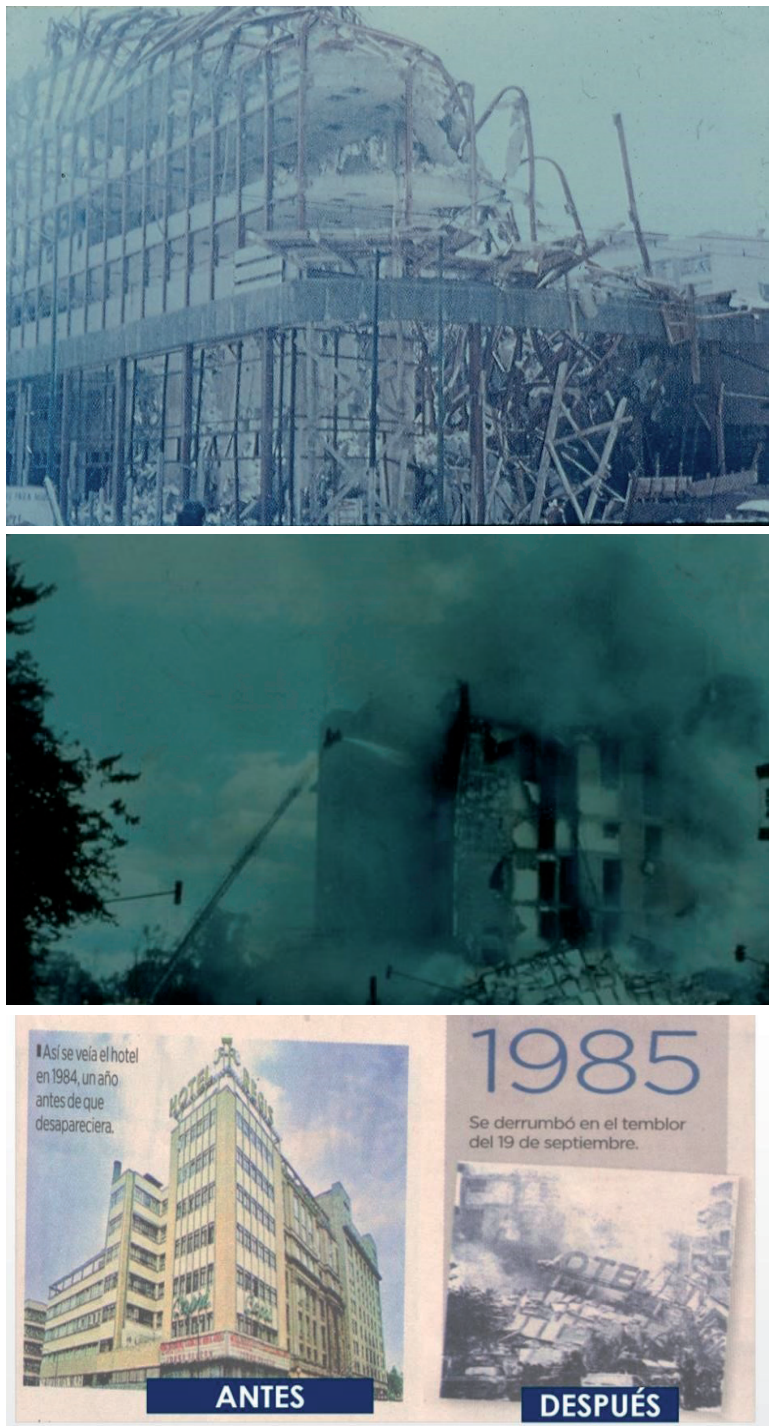

Figura 1. 
La Torre Latinoamericana, que tiene 55 niveles y pesa unas 25000 toneladas, situada en pleno Centro Histórico de la ciudad de México, no mostraba ningún daño, situación similar se tuvo en algunos edificios de la época colonial.
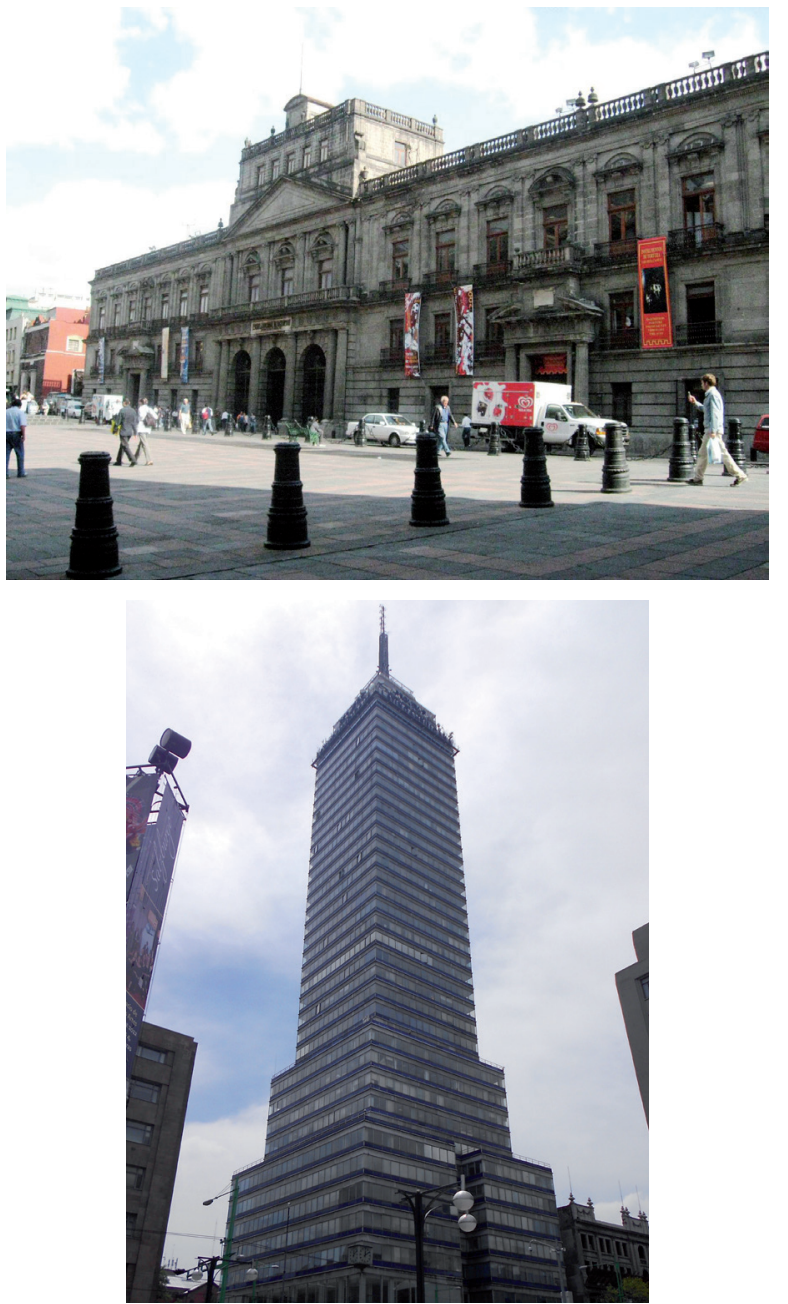

Figura 2.

En la zona centro el sismo tuvo un periodo del orden de 1.5 segundos y los edificios colapsaron, en general podría considerarse que habían sido diseñados y construidos de tal forma que su periodo tenía una magnitud similar, este hecho llevó a la conclusión que en ellos, durante el sismo, se generó el fenómeno denominado "resonancia" en que los movimientos del suelo de apoyo provocados por el sismo, se suman a los que en un instante experimentaron las estructuras de los edificios dañados, generando con ello esfuerzos en las mencionadas estructuras que sobrepasaron a aquellos debido a la "resistencia".

Este fenómeno no ocurrió en la Torre Latinoamericana debido a que su periodo fue del orden de los 5.5 segundos, magnitud muy diferente al generado por el sismo en el suelo de la zona.

En cuanto a los edificios localizados en esquinas, se encontró que su diseño arquitectónico los dotó de paredes hacia las calles cubiertas de vidrios y una estructura propicia a sostenerlos, mientras que las paredes hacia el interior eran de mampostería, columnas y trabes poco flexibles, ello generó que la rigidez en estos últimos fuese bastante mayor que la de las paredes externas; está no uniformidad de rigidez propició que al ocurrir el sismo se produjese tensión en los edificios, lo que los colapsó.

El hecho de tener, desde el punto de vista principalmente geotécnico, formaciones naturales en donde el riesgo sísmico fue mayor implicó proceder a microregionalizar el suelo de la ciudad de México y a modificar, en lo conducente, el Reglamento de Construcción y para ello hubo que considerar los factores que influyen en el llamado riesgo sísmico.

\section{INFLUENCIA DE LAS CONDICIONES LOCALES DE LOS SUELOS EN EL RIESGO SÍSMICO}

Varios son los factores que se conjugan para determinar las consecuencias de un sismo. Sin embargo, hay uno que pertenece a la geotecnia y que aún no recibe, en la práctica, la atención que merece. Se trata de la influencia de las condiciones locales de los suelos en los efectos sísmicos. Su estudio permite explicar, entre otras cosas, por qué los daños provocados por un sismo en una misma zona y sobre estructuras similares, son diferentes. Pero su mayor trascendencia radica en que la debida atención de este aspecto permitirá ahorrar enormes sumas en daños materiales y pérdida de vidas en las zonas urbanas de alta sismicidad, mediante la estimación cuantitativa del efecto de las formaciones de suelo sobre las solicitaciones sísmicas.

Elriesgo sísmico es un fenómeno complicado, dada la diversidad de factores que intervienen y la complejidad con que lo hacen; por ello los investigadores han considerado conveniente enfrentarlo en tres niveles. El primero geológico, en que habrán de tomarse en cuenta las características geológicas generales de la zona en que se pretenda predecir la probabilidad de ocurrencia de sismo; el segundo a nivel geotécnico, en el que intervendrán las características topográficas, estratigráficas y mecánicas de las formaciones de suelos existentes en cierto sitio, a fin de definir las características que un sistema adquirirá en ella; y el tercer nivel, estructural, en términos de su efecto en obras específicas, 
en que la meta será definir las consecuencias que los sismos característicos del sitio tendrán en las estructuras. En Ingeniería Sísmica se usa el nombre "riesgo sísmico total" para indicar aquel en que se conjuntan los riesgos según los tres niveles mencionados (1).

Desde el punto de vista geológico puede afirmarse que los sismos más importantes o más frecuentes se generan a lo largo de fallas geológicas de la corteza terrestre donde las presiones internas del magma más próximo a la corteza terrestre activan el desplazamiento de estas fallas; así, en el caso de la república mexicana los epicentros más importantes (zonas generadoras de sismos), están situados en la costa del Pacífico (igura 3). Precisamente en la zona correspondiente a una de las fronteras de la placa tectónica denominada Cocos; circunstancialmente resulta conveniente comentar que la teoría de las placas tectónicas ha permitido tener un panorama más amplio respecto a los sitios de la tierra que se considera tienen un mayor potencial como posibles fuentes generadoras de sismos (2); y con relación a estas zonas, se puede pensar que aquellas que se encuentran en movimiento relativamente continuo no permiten la acumulación de energía en magnitud tan grande como la que se genera en una falla cuyo desplazamiento relativo es muy pequeño o no existe, de manera que, en términos generales, serán estas últimas las que permitan la generación de los sismos de mayor magnitud.

Investigadores del Instituto de Ingeniería de la Universidad Nacional Autónoma de México, han dedicado su atención al estudio del riesgo sísmico; entre los factores que se han considerado están las características geológicas y los
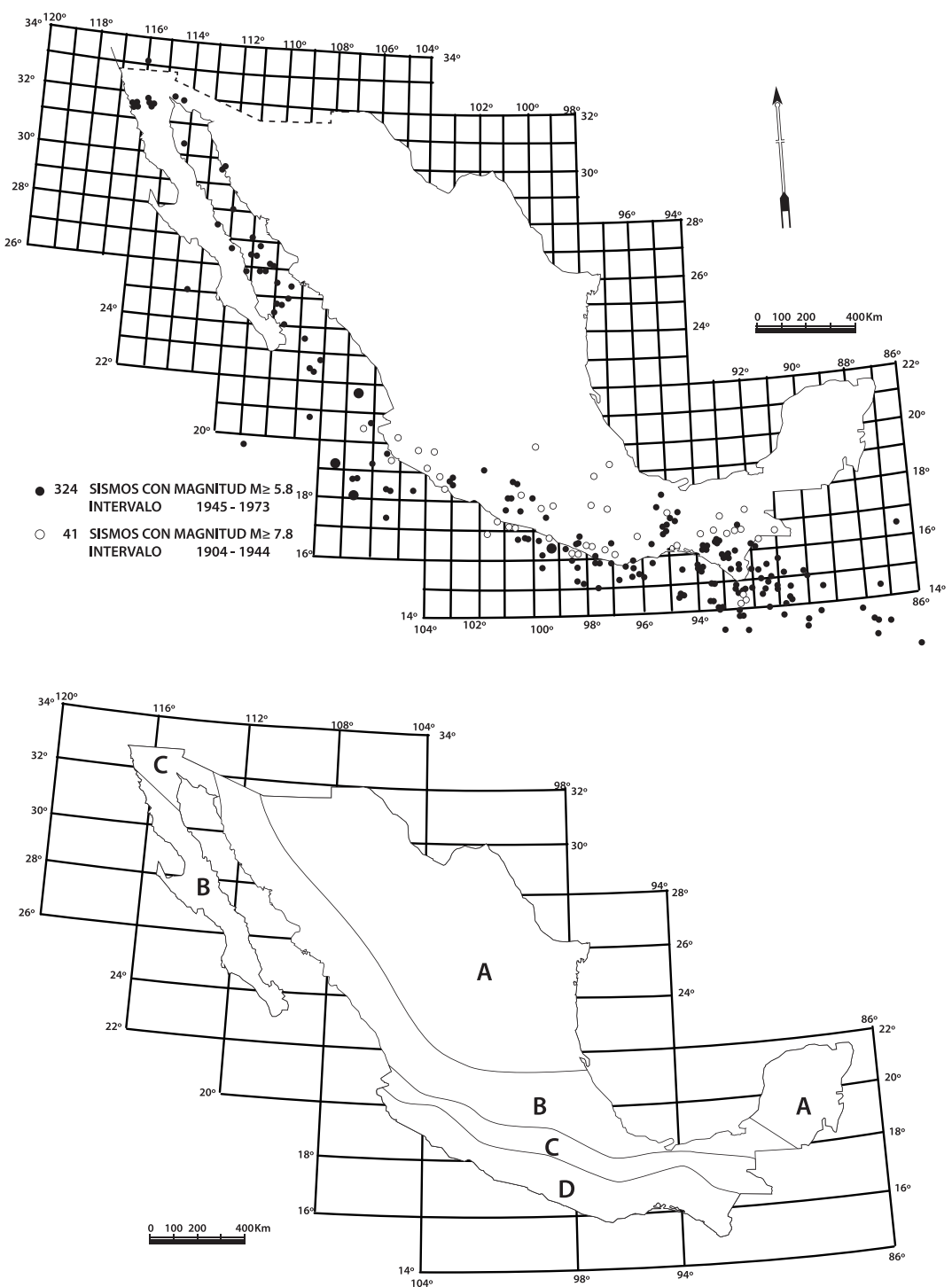

Figura 3. Regionalización sísmica de México (Ref. 4). 


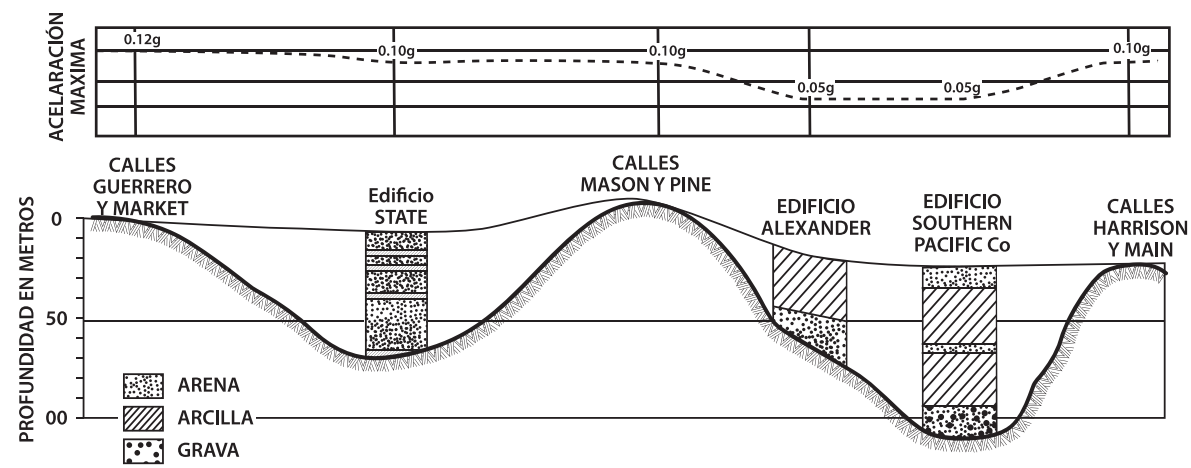

Figura 4. Características de los suelos y aceleraciones máximas en el sismo registrado en San Francisco (E.U.A.) en 1957 (Ref. 5).

suelos existentes en la zona en donde se trata de evaluar el riesgo.

En la figura 4, el riesgo sísmico se incrementa de las zonas marcadas con A a la indicada con $\mathrm{D}$.

Aparentemente, desde el siglo XVIII y como consecuencia del sismo de Lisboa (1755), se tuvieron las primeras observaciones acerca de la influencia de las características de los suelos en los efectos de los sismos. En la literatura (57) se indica que ya en los reportes escritos del sismo de San Francisco, en 1906, se menciona el efecto de las condiciones de los suelos locales en los daños. Pero es hasta el sismo de Kanto en 1923, en que sufrieron daños un total de 128266 edificios (61) destruyéndose la ciudad de Tokyo y sus alrededores, en que se detalla la correlación. Una posible explicación de este hecho puede hacerse a través de la consideración de las características de los suelos existentes en los lugares en que se tuvieron instrumentos registradores.

Seed e Idriss, (7), Rizada, Nakagawae Izumi (8), muestran una serie de espectros de respuesta sísmica; cuatro corresponden a movimientos registrados en la misma ciudad, durante el mismo sismo; los otros dos corresponden a sismos de magnitudes similares, y todos se obtuvieron en sitios bastante alejados de los epicentros.

En la figura 4 se ilustran los espectros; se indican en ella las características de los suelos que aparecen en los lugares de medición; los espectros se han ordenado de manera que al pasar de uno a otro la rigidez del perfil de suelos en cada sitio va disminuyendo relativamente, al ir apareciendo espesores mayores de suelos finos. De la figura se tiene que a medida que la rigidez de los suelos va disminuyendo el período en que se presenta la aceleración máxima aumenta, tal como indica la siguiente tabla:
TABLA 1

\begin{tabular}{|c|c|}
\hline LUGAR DE MEDICIÓN & $\begin{array}{l}\text { PERÍODO EN EL QUE SE DESARROLLA LA } \\
\text { ACELERACIÓN MÁXIMA }\end{array}$ \\
\hline A & 0.3 segundos \\
\hline B & 0.5 segundos \\
\hline C & 0.6 segundos \\
\hline D & 0.8 segundos \\
\hline$E$ & 1.3 segundos \\
\hline $\mathrm{F}$ & 2.5 segundos \\
\hline
\end{tabular}

Los datos de la tabla, parecen señalar la tendencia comentada, pero es obvio que lo que interesa son los probables daños que el sismo de que se trate pudieran causar, y a este respecto se puede pensar que la probabilidad de mayor daño en estructuras que existan en el sitio, ocurrirá cuando el periodo natural de vibración de la estructura iguale al del terreno.

Durante el sismo del 28 de julio de 1957 en ciudad de México, la aceleración máxima, no tuvo magnitudes extraordinarias.

En el centro de la ciudad, se estimaron valores de $0.05 \mathrm{~g}$ a $0.10 \mathrm{~g}$, pero en cambio sus características de frecuencia y duración fueron suficientes para que la coincidencia mencionada ocurriera y así causar daños considerables en estructuras altas $(9,10)$. En cambio, en Junio de 1966; se produjo un sismo en la zona de Park Field, en el Estado de California, Estados Unidos. La aceleración máxima del terreno fue de $0.5 \mathrm{~g}$ pero el periodo fundamental de vibración fue muy pequeño, lo mismo la duración del sismo, y puede pensarse que, consecuentemente, la coincidencia mencionada no ocurrió y los daños causados a estructuras altas fueron pequeños (11).

Por lo tanto, volviendo a los datos consignados en la figura 4 y en la tabla, se tiene que en los perfiles de suelos rígidos la aceleración máxima tiende a suceder para periodos relativamente pequeños de 0.4 a 0.5 segundos, lo que pudiera indicar que en esos sitios las máximas 
aceleraciones serán inducidas en estructuras relativamente rígidas, por ejemplo de menos de 5 ó 6 niveles con estructuración común. En cambio, en los perfiles de suelos más flexibles, el periodo fundamental es relativamente grande, de 1.5 a 2.5 segundos, lo que a su vez puede interpretarse como que en esos sitios, la probabilidad de que ocurran daños será mayor en estructuras altas, por ejemplo del orden de 20 a 30 niveles.

La rigidez del perfil de suelos no sólo depende de las características de compacidad, en los suelos gruesos, o de consistencia en el caso de los suelos finos, sino que también influye el espesor. Al respecto, se tiene el caso del sismo que ocurrió el 29 de julio de 1967 en la ciudad de Caracas y sus alrededores (12).
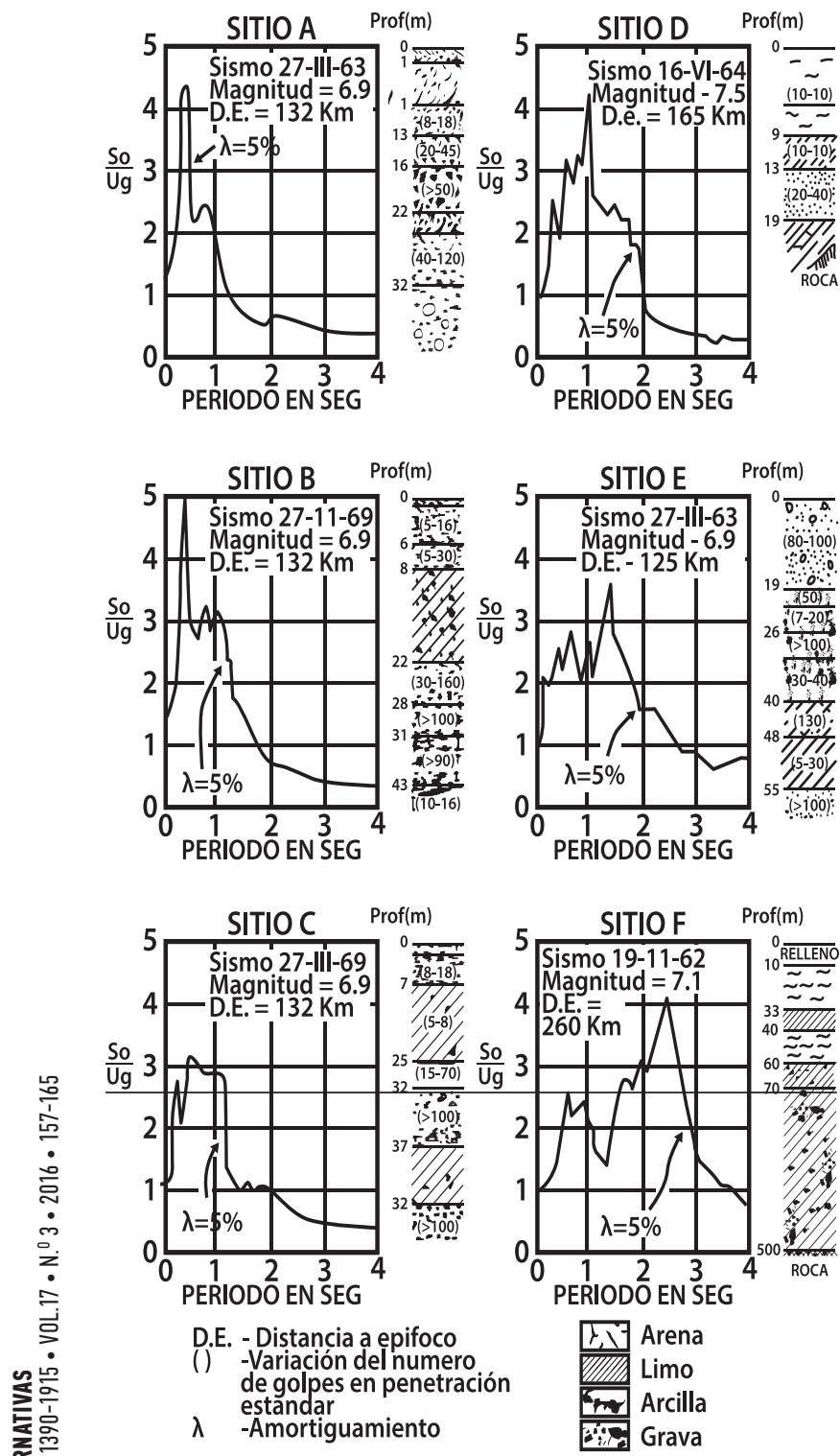

Figura 5. Efecto de las características del suelo sobre la forma del espectro respuesta. (Ref. 7 y 8 ).
La magnitud del sismo fue de 6.4 y su epicentro se localizó a unos 55 kilómetros de Caracas. (figura 5). La ciudad de Caracas está localizada a unos 16 kilómetros de la costa noreste de Venezuela en un valle formado por depósitos aluviales y cruzado por el río Guaire de oeste a este.

Los suelos que forman el valle pueden $\mathrm{ca}^{-}$ lificarse cualitativamente como buenos para apoyar en ellos estructuras; están constituidos por arenas, gravas y algunas capas de arcilla dura. Es interesante anotar que, de acuerdo con los estudios realizados, las características dinámicas de los suelos no varían mucho a lo largo del valle, no así sus espesores según lo indica la figura 7, en que se observan los resultados obtenidos mediante un estudio geofísico.

La figura 7 muestra que los daños se concentraron en el lado este de Caracas, en la zona denominada Palos Grandes, en que colapsaron cuatro edificios, de 10 a 12 niveles (200 vidas perdidas) y resultaron dañados estructuralmente casi todos los edificios de más de 14 niveles, siendo definitivamente menor el daño en estructuras de poca altura.

En cambio en el noreste, el daño fue considerablemente alto en estructuras de uno y dos niveles; el daño fue menor en estructuras de más de 10 niveles. Los edificios dañados con número de niveles entre 6 y 9 estuvieron más o menos distribuidos en toda el área de la ciudad. A lo largo de la costa el daño fue particularmente severo en Caraballeda, localizada en un abanico aluvial, constituido por arenas con intercalaciones de capas de limo y arcilla que en la línea de la costa alcanzan espesores del orden de los 90 metros, bajo ellos aparece una pizarra con un espesor muy potente. En esa zona sufrieron daño total los cuatro pisos superiores de un edificio de once niveles, además de que otros edificios sufrieron daño estructural.

Como no había razón para creer que la calidad del diseño y la construcción de los edificios fuera diferente en Palos Grandes o en Caraballeda, respecto a lo que se tuvo en otras partes de Caracas, de la línea de costa, el estudio que se realizó se enfocó a encontrar otras posibles causas de esas concentraciones de daños, concluyendo que esto podría ser básicamente el espesor de los suelos que aparecieron en esas zonas.

Aún cuando los resultados anotados son valederos para las características del sismo de 1967, y en la zona de Caracas, ellos ponen de manifiesto la influencia notable que el espesor de suelo tuvo en los efectos del sismo. 


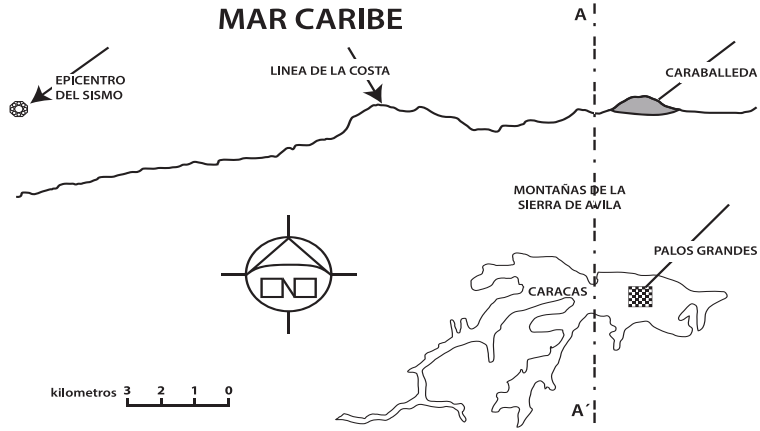

Figura 5. Mapa de Caracas. (Ref. 12).

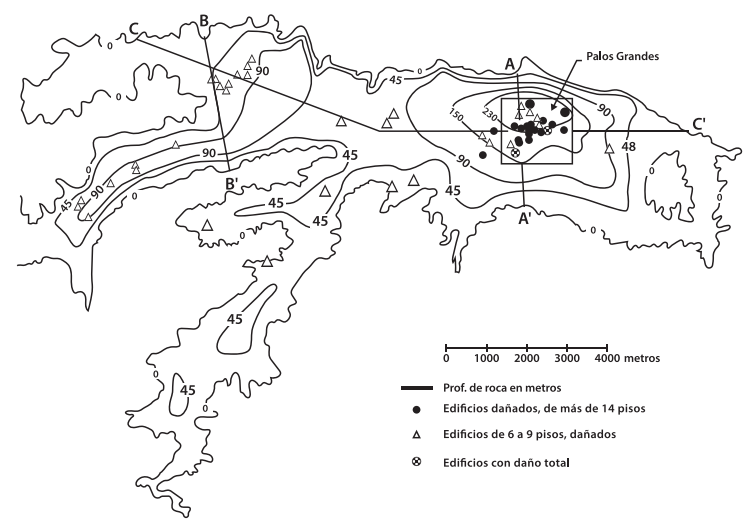

Figura 7. (Ref.12).

Las características topográficas de los depósitos de suelos, parecen también influir en los efectos de los sismos; existen pocos estudios y reportes al respecto, de manera que no es posible hacer conclusiones definitivas; este hecho conduce a que sea necesario que se esté atento a reportar y estudiar en forma acuciosa los efectos sísmicos ligados a esta característica.

De acuerdo con las observaciones hechas de los efectos provocados por diferentes sismos ocurridos en la zona de la ciudad japonesa Nagoya, se sabe que los mayores destrozos han sucedido siempre en la parte baja de la misma. En el año de 1944, se produjo, en la zona, el sismo Tonankai, y en el estudio hecho por Omote y Miyamura (13) aparece una gráfica que cuantifica lo indicado antes. Sin embargo, se indica que este resultado no puede ser concluyente en cuanto a la influencia de la topografía, ni aún para la zona, porque la compacidad y la consistencia de los suelos existentes no son uniformes; estas características son bastante mejores en las partes altas que en las bajas.

Se han intentado algunos análisis teóricos, mediante el método del elemento finito, para casos particulares de taludes. En la figura 9 se muestran las aceleraciones máximas horizontales y verticales en la superficie de un estrato de arcilla, de 15 metros de altura, lateralmente

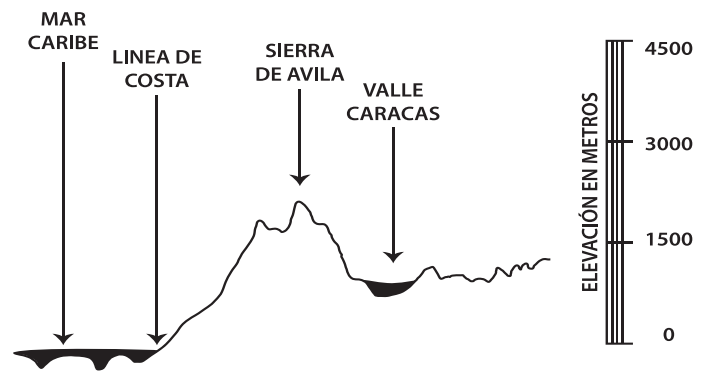

Figura 6. Sección A-A' (Ref. 12).

limitado por un talud ( dos horizontal y uno vertical), que sobrayace a un manto de roca horizontal, con espesor también de 15 metros, a la que se sometió a una aceleración máxima de $0.3 g$ (14).

De acuerdo con los resultados teóricos, las máximas aceleraciones se desarrollan en la inmediata proximidad del hombro del talud.

La presencia de suelos granulares hace que los efectos de los sismos se traduzcan en asentamientos rápidos y en disminución brusca de la resistencia al esfuerzo cortante.

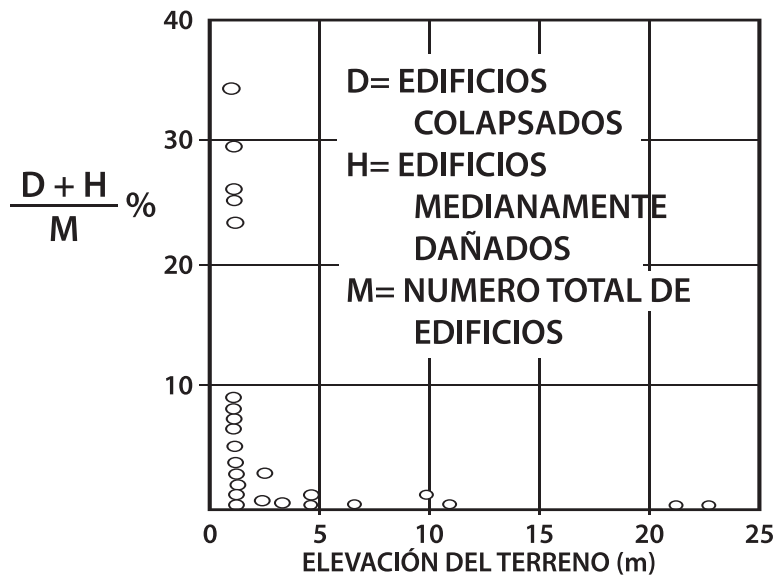

Figura 8. Elevación del terreno/daño a edificios de madera, en Nagoya. Sismo Tonankai 1944 (Ref. 13).

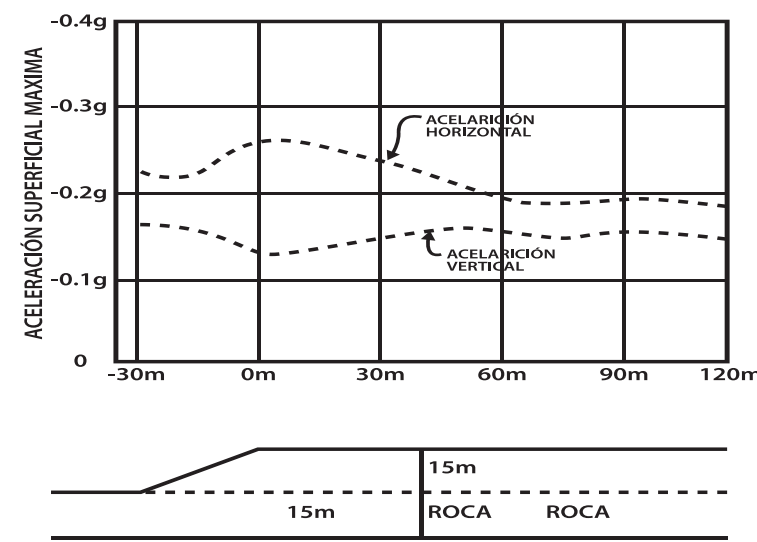

Figura 9. Aceleraciones máximas en la superficie del suelo, limitado por un talud. (Ref. 14). 
La disminución brusca de la resistencia de los suelos básicamente granulares saturados o secos puede llegar al extremo de provocar su licuación; este fenómeno ha sido la causa de que sismos de características poco importantes hayan generado pérdidas considerables.

Existen y se realizan actualmente numerosas investigaciones al respecto. Ellas han puesto de manifiesto que los factores importantes que influyen en la ocurrencia de la licuación pueden ser:

1. Tipo de suelo.

2. Compacidad relativa del suelo.

3. Esfuerzo confinante inicial.

4. Magnitud del esfuerzo repetido.

5. Número de ciclos de esfuerzo.

El Gobierno Federal de la República Mexicana inició estudios que tienen como meta final la regionalización sísmica y geotécnica de las áreas urbanas y futuras del país. El estudio se ha iniciado seleccionando las ciudades del país que se considera deben tener un crecimiento urbano futuro más importante. A la fecha se han hecho estudios preliminares en 32 ciudades. En cada uno de los estudios, se va de lo general a lo particular; así, se analizan las características correspondientes a la Geología Regional

\section{REFERENCIAS BIBLIOGRÁFICAS}

1. Whitman R. V. y C. A. Cornell.- Capitulo IX del libro Seismic Risk and Engineering Decisions por C. Lomnitz y E. Rosenblueth.- Elsevier Scientific Publishing CompanY. (1976).

2. C. Lomnitz.- Global Tectonics and Earthquake Risk. Elsevier, 1974.

3. Esteva Luis.- Capitulo VI del libro Seismic Risk and Engineering Decisions por Lomnitz y E. Rosenblueth- Elsevier Scientific Publishing Company. (1976)III-6512.

4. Trigos José Luis.- Regionalización Sísmica.- Articulo preparado para Secretaria de Asentamientos Humanos y Obras Públicas.-(1975).

5. Wood, H.O. "Distribution of Apparent Intensity in San Francisco, in the California Earthquake of Abril 18, 1906 ". Report of the State Earthquake Investigation Lomission, Carnegie Institution of Washington, Washington, D.C.

6. Ohaaki, Y., 1969.- "Effects of Local Soil Conditions upon Earthquake Damage" Proc. Soil Dynamics Specialty Sessión, Int. Conf. SoilMech. and Foundation Engrg. Woodword Lundgren Assoc. Oakland,Calif. U. Luscher, ed.

7. Seed, H. Bolton, and Idriss, I. M."Influence of Soil Conditions on Ground Motions during a la Geotecnia, incluyendo las fisiográficas y estratigráficas generales de la zona; especial atención se le da a la geología estructural y a la histórica y desde luego a los aspectos sísmicos.

\section{CONCLUSIONES Y RECOMENDACIONES}

a. Es pertinente, en el diseño de estructuras, el considerar el no generar acciones relacionadas con el fenómeno de resonancia, además de unificar la rigidez de la estructura.

b. De acuerdo con lo comentado, es innegable que el conocimiento de las características mecánicas y estratigráficas de los suelos en la zona en que se pretenda valuar el llamado riesgo sísmico, adquiere una importancia fundamental que no se debe soslayar.

c. Es evidente que es urgente el que todas las áreas urbanas actuales, y también las futuras, que se localizan o localicen en zona sísmica se microzonifiquen desde el punto de vista de la respuesta sísmica esperada del perfil de suelos.

d. Es necesario que los planes de desarrollo urbano tomen en cuenta en forma importante, los resultados de la microzonificación.

Earthquakes: Journal of the Soil Mechanics and Foundations Division, ASCE, Vol.95, Ng SM1, pp. 99-137. Enero (1969).

8. Hizada, T., Nakagawa, K. e Izumi.-Normalized Acceleration Spectra for Earthquakes Recorded by Strong Motions Accelerographs and their Characteristics Related with Subsoil Conditions ", BRI Report Ns 2 ,Building Research Institute, Ministry of Construction, Tokyo, Japón, (1965).

9. Merino y Corchado, J. "El temblor del 28 de Julio de 1957" Anales del Instituto de Geofísica de la Universidad Nacional Autónoma de México. (1957).

10. Rosenblueth, E. "The Earthquake of 28 July 1957 in Mexico City". Memorias de la 4. Conferencia Internacional de Ingeniería Sísmica, Japón, Volumen I. (1960).

11. Cloud, W. K. "Intensity Map and Structural Damage, Parkfiel.d, California, Earthquake of June 27,1966". Boletín de la Sociedad Sismológica de América. Vol. 57 Ng 67(1967).

12. Seed B., Whitman R., Houshang Dezfulian, Dobry R., Idriss I. M. - "Soil Conditions and Building Damage in 1967 Caracas Earthquake" Journal of the Soil Mechanics and Foundations Division. Proceedings of the American Society of Civil Engineers. (1972). 
13. Omete, S. y S. Miyamura. "Relations between the Earthquake Damage and the Structure of Ground in Nagoya_ City, Bulletin of the Earthquake Research Institute, Universidad de Tokyo, (1951).

14. Idriss, I. M. Seed, H. B. "Response of Earth Banks During Earthquakes". Journal of the Soil Mechamica and Foundations Division, ASCE Vol. 93, NQ SM3, May, pp Sr82. (1967).

15. Retamal, Eugenio y Kausel, Edgar."Vibratory Compaction of the Soil and Tectonic Subsidence During the1960 Earthquake in Valdivia, Chile ". Proceedings, Fourth World Conference of Earthquake Engineering, Santiago, Chile, Enero, (1969).

16. Whitman, R. V., y Ortigosa, P.,"Densification of sand by vertic vibrations" Technical paper T68 5 . Dept. of Civil Engrg, Massachússetts, Institute of Technology, Cambridge, Maasachussetts, (1968)7.
17. Silver, M. L. y Seed, H. B., "The behavior of sands under seismic loading conditions. Report EERC 69 - 16 Earthq. Engrg. Res. Center Universidad de California, Berkeley, California, (1969).

18. Youd, T. L. "Densification andshear of sand during vibration". Proc. ASCE, 96 (SM3). 863 - 880. (1970).

19. Seed, H. B. e Idriss, I. M. "A simplified procedure for evaluating soilliquefaction potential". ReportEERC 70 - 9, Earthq. Engrg. Res. Center, Universidad de California, Berkeley, California. (1970b).

20. Ohsaki, Y., "Niigata earthquakes, 1964, building damage and condition Soil and Foundations ", 6 (27714 - 37 (1966).

21. Seed, H. B., and Lee, K. L. Liquafaction of saturated sands during_cyclic loading. Proc. ASCE, 92 (SM6): 105 - 134 (1966). 